\title{
Progress of Picrotoxane Type Alkaloids in Dendrobium
}

\author{
Jingjuan $\mathrm{Li}^{a, b, c}$ Fucai Ren, ${ }^{a, b, c}$ and Jiangmiao $\mathrm{Hu}^{*, a, b}$ \\ a State Key Laboratory of Phytochemistry and Plant Resources in West China, Kunming Institute of Botany, \\ Chinese Academy of Sciences, Kunming, Yunnan 650201, China \\ ${ }^{b}$ Yunnan Key Laboratory of Natural Medicinal Chemistry, Kunming Institute of Botany, \\ Chinese Academy of Sciences, Kunming, Yunnan 650201, China \\ ${ }^{c}$ School of Chemical Sciences and Technology of Yunnan University, Kunming, Yunnan 650091, China
}

Email: hujiangmiao@mail.kib.ac.cn (J. H.)

\begin{abstract}
Dendrobium has a long clinical usage history and diverse chemical compositions. The picrotoxane type alkaloid represented by dendrobine from Dendrobium species has antipyretic and anti-hypertensive activities. Since the 1930s, the study of dendrobine has made a huge progress, including many new reported picrotoxane type alkaloid compounds. In this article, some developments of picrotoxane type alkaloids in $D$. wardianum and $D$. findlayanum are described.
\end{abstract}

Keywords Dendrobium, picrotoxane type alkaloid, dendrobine, wardianumine A, findlayines

Picrotoxane type alkaloids, also called dendrobine-type alkaloid, only distribute in some species of Dendrobium. Up to now, 27 picrotoxane type alkaloids have been reported from 8 species of Dendrobium (Figure 1).

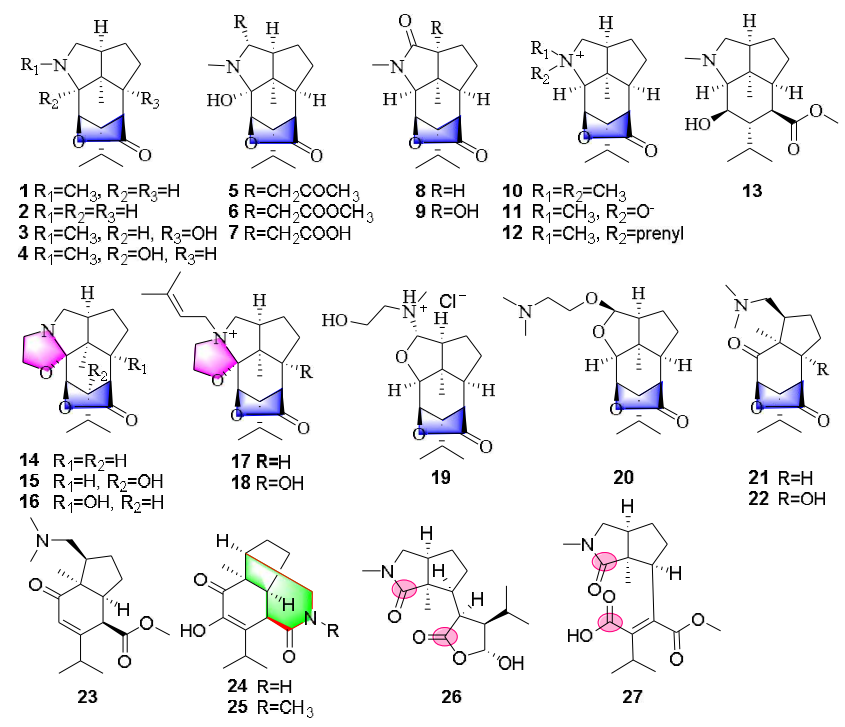

Figure 1 Reported picrotoxane type alkaloids in Dendrobium.

The first isolation of dendrobine from $D$. nobile was done by Hideki Suzukiand in 1932, then Chen Ke-Hui also got the compound in 1935..$^{[1,2]}$ The sesquiterpenoid structural skeleton of dendrobine was not elucidated until that was solved by Tadamasa Onaka et al. in $1964,{ }^{[3]}$ which marked the beginning of the systematic research of picrotoxane type alkaloids. Due to antipyretic and anti-hypertensive activities of this kind alkaloid and 7 contiguous stereocenters, dendrobine has become a research hotspot for organic chemists. A series of total synthesis were published in many journals and (-)-dendrobine was synthesised by Diels-Alder reaction recently with a high stereoselectivity. ${ }^{[4-15]}$

Dendrobine-type alkaloids mainly distributed in sect.
Dendrobium of Dendrobium species, however, few has been reported recently. ${ }^{[16]}$ Since a long-term chemical research focused on Dendrobium and analysis comparison of different species, we can now ascertain which species from Dendrobium contain dendrobine-type alkaloids efficiently. As a result, one dendramine, named wardianumine $\mathrm{A}$, was isolated from $D$. wardianum (20 in Figure 1 ) by us ${ }^{[17]}$ and it provides an idea for the synthesis pathway of dendrobine. Furthermore, four novel seco-dendrobine-type alkaloids named findlayines $A-D$ (Figure 1, 24-27) were isolated fortunately by us from $D$. findlayanum, and the results enriched the diversity of picrotoxane type alkaloids. ${ }^{[18]}$

Chemical studies on four new picrotoxane type alkaloids showed that two compounds (Findlayines $A$ and $B$ ) with a 5/6/7 tricyclic cage skeleton and two compounds (Findlayines $\mathrm{C}$ and D) derived by the cleavage of the $C_{2}-C_{3}$, which is rare in this type of alkaloid. In addition, the absolute configurations of 24-27 were confirmed by single crystal X-ray diffraction (Figure 2). ${ }^{[18]}$

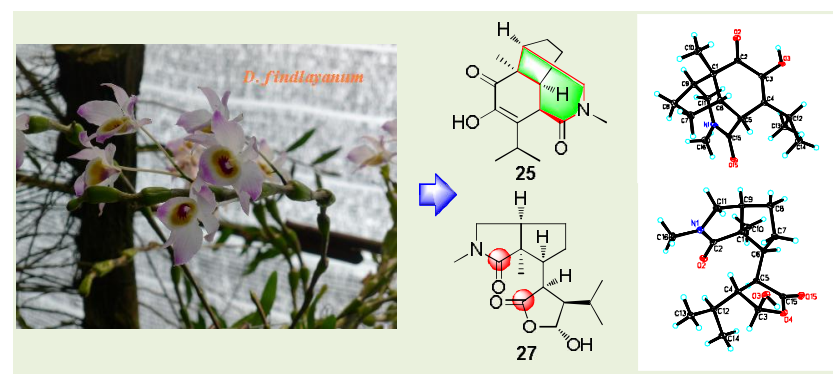

Figure 2 Chemical structures of findlayines $A$ and $C$.

\section{References}

[1] Suzuki, H.; Keimatsu, I. Yakugaku Zasshi 1932, 52, 1049.

[2] Chen, K. H.; Chen, A. L. J. Biol. Chem. 1935, 111, 653.

[3] Onaka, T.; Kamata, S.; Maeda, T.; Kawazoe, Y.; Natsume, M.; Okamoto, T.; Shimizu, M. Chem. Pharm. Bull. 1964, 12, 506.

[4] Yamada, K.; Suzuki, M.; Hayakawa, Y.; Aoki, K.; Nakamura, H.; Nagase, H.; Hirata, Y. J. Am. Chem. Soc. 1972, 94, 8278.

[5] Kende, A. S.; Bentley, T. J.; Mader, R. A.; Ridge, D. J. Am. Chem. 
Soc. 1974, 96, 4332.

[6] Roush, W. R. J. Am. Chem. Soc. 1978, 100, 3599

[7] Trost, B. M.; Tasker, A. S.; Ruther, G.; Brandes, A. J. Am. Chem. Soc. 1991, 113, 670.

[8] Lee, C. H.; Westling, M.; Livinghouse, T.; Williams, A. C. J. Am. Chem. Soc. 1992, 114, 4089.

[9] Uesaka, N.; Saitoh, F.; Mori, M.; Shibasaki, M.; Okamura, K.; Date, T. J. Org. Chem. 1994, 59, 5633.

[10] Sha, C. K.; Chiu, R. T.; Yang, C. F.; Yao, N. T.; Tseng, W. H.; Liao, F. L.; Wang, S. L. J. Am. Chem. Soc. 1997, 119, 4130.

[11] Cassayre, J.; Zard, S. Z. J. Am. Chem. Soc. 1999, 121, 6072.

[12] Hu, Q. Y.; Zhou, G.; Corey, E. J. J. Am. Chem. Soc. 2004, 126, 13708.

[13] Kreis, L. M.; Carreira, E. M. Angew. Chem. Int. Ed. 2012, 51, 3436.
[14] Nawrat, C. C.; Moody, C. J. Angew. Chem. Int. Ed. 2014, 53, 2056

[15] Guo, L.; Frey, W.; Plietker, B. Org. Lett. 2018, 20, 4328.

[16] Ye, Q. H.; Zhao, W. M.; Qin, G. W. In The Progress in Medicinal Chemistry, Vol. 3, Ed.: Peng, S. X. Chemical Industry Press, Beijing, 2002, p. 113.

[17] Zhang, C.; Liu, S. J.; uan, M. Y.; Li, J. Y.; Hou, B.; Li, H. M.; Yang, X. Z.; Ding, C. C.; Hu, J. M. Fitoterapia 2017, 122, 76.

[18] Yang, D.; Cheng, Z. Q.; Yang, L., Hou, B.; Yang, J.; Li, X. N.; Zi, C. T.; Dong, F. W.; Liu, Z. H.; Zhou, J.; Ding, Z. T.; Hu, J. M. J. Nat. Prod. 2018, 81, 227

Received June 29, 2019 Accepted July 16, 2019 